\title{
6Genesis Flagship Program: Building the Bridges towards 6G-enabled Wireless Smart Society and Ecosystem
}

\author{
Marcos Katz \\ Centre for Wireless Communications \\ University of Oulu \\ Oulu, Finland \\ marcos.katz@oulu.fi
}

\author{
Marja Matinmikko-Blue \\ Centre for Wireless Communications \\ University of Oulu \\ Oulu, Finland \\ marja.matinmikko@oulu.fi
}

\author{
Matti Latva-aho \\ Centre for Wireless Communications \\ University of Oulu \\ Oulu, Finland \\ matti.latva-aho@oulu.fi
}

\begin{abstract}
In this paper, an initial vision of what the sixth generation mobile communication system (6G) might be is presented. This is done through the primary ideas of the 6Genesis Flagship Program (6GFP), a recently formed Finnish academic and industrial consortium aiming at developing key enabling technologies for 6G. 6GFP is an eight-year large-scale research initiative set to ultimately develop, implement and test key enabling technologies for $6 \mathrm{G}$. Motivations, visions, trends and expectations for $6 \mathrm{G}$ are first discussed to set the ground for further development. Four main research areas are proposed as the starting point, encompassing not only wireless communications but also computer science, electronics and material science as well as applications and services. The paper provides an in-detail list of technical topics going to be studied in this program, and in general, worth considering for everyone planning to develop beyond 5G technology. The paper also shed some light on how the research will be implemented, showing the logic and relationships between the research areas, discussing already selected promising enabling technology for 6G, as well as envisioning how the proof-of-concept of the developed system will be carried out. An existing state-of-theart $5 \mathrm{G}$ network will be used as the starting point for developing and testing the novel concepts and technologies created in the program (Abstract)
\end{abstract}

Keywords - 6G, Beyond $5 G$, future wireless and mobile networks, framework project, mobile communications; proof-of concept, mm-wave, tera-Hz bands (Keywords)

\section{INTRODUCTION}

Looking back at the evolution of mobile communications over the past decades, one can see that there are similar development cycles related to each generation $(G)$. Typically, developing a new mobile generation takes one decade, while its further usage extends for at least another decade. The developing process includes definition, specification, standardization and industrial implementation of the technology. Academy, research organizations, industry and regulators cooperate on a global basis to develop a new generation of mobile communications. As $5 \mathrm{G}$ is currently in the final phase of developing and close to its massive commercialization, expected to begin approximately in 2020 , the research and industrial communities are starting to concentrate in the development of the sixth generation $(6 \mathrm{G})$ mobile communication system. Launching of $6 \mathrm{G}$, including commercialization and deployment, is expected to occur around 2030, preceded by a decade of development. In this paper, we introduce the 6Genesis Flagship program, an allencompassing Finnish initiative focused on the development of a complete $6 \mathrm{G}$ ecosystem. The key goals of the $6 \mathrm{Genesis}$
Flagship Program (6GFP) include supporting industry in the final development of $5 \mathrm{G}$, developing fundamental enabling technologies for $6 \mathrm{G}$ and accelerating the process of digitalization of society. This large-scale research program has a rather broad scope, in three main areas, namely wireless communications, computer science and engineering, and electronics and materials, as depicted in Figure 1. The overall volume of the $6 \mathrm{G}$ flagship program will be approximately $\$ 290$ million over eight years.

This paper presents the 6GFP's visions on the upcoming $6 \mathrm{G}$, identifying relevant research topics as well as presenting some of the lines of research to be followed in order to achieve the aforementioned goals. The paper intends also to serve as a motivational technical guide, identifying relevant research topics and promising concepts and techniques with potential to become key technology enablers of $6 \mathrm{G}$. It is expected that the insights presented here will further foster the development of novel ideas, concepts and technologies to be exploited by $6 \mathrm{G}$.

This paper is organized as follows. Section II provides a number of important visions for 2030. Then, Section III describes the four main research and development areas. The lines of research needed to achieve the program goals are described in Section IV. Finally, Section V concludes this paper.

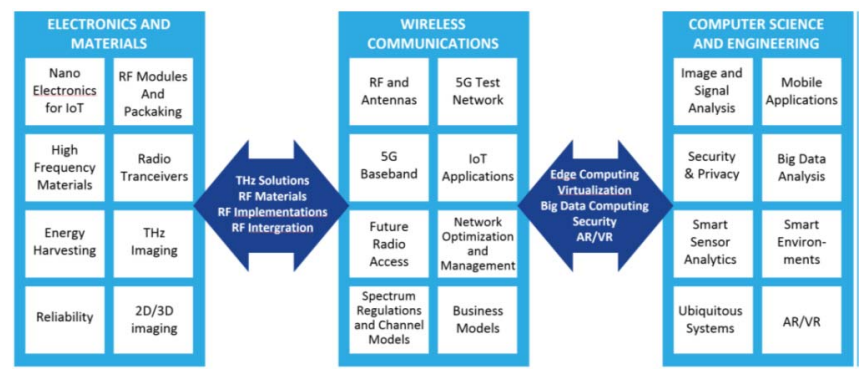

Figure 1.Towards 6G: Three research areas considered by 6GFP.

\section{TOWARDS 2030: VISIONS AND TRENDS}

As mobile and wireless communications grow in terms of data volume, number of users and investment in communications infrastructure, users expect better and more versatile services, while network operators expect increasingly higher profits. To achieve these goals, the research community needs to continuously improve link and network performance as well as enhance utilization of radio 
resources, particularly energy and spectrum. From the first generation $(1 \mathrm{G})$ until the current ones (4G and $5 \mathrm{G})$, there has been a gradual and substantial shift toward the use of higher, less congested frequencies bands. This is also the case for $6 \mathrm{G}$, where frequencies from $100 \mathrm{GHz}$ to $\mathrm{THz}$ are being considered. This gigantic jump in the frequency domain from current fey $\mathrm{GHz}$ all the way up to the $\mathrm{THz}$ band requires reengineering of virtually the whole system. Devices, transceivers and network-related technologies, among others, need to be designed and optimized for these very-high frequency bands. Novel services are also expected to put stringiest requirements on network reliability, latency, security as well as on capacity. More than ever, users expect an omnipotent and omnipresent yet invisible communication network supporting them whenever needed. With an expected very-high wireless device density (e.g., smartphones, tablets, appliances, sensors, etc.) a massive amount of data need to be efficiently and timely processed. This would require novel data management techniques exploiting distributed intelligence and artificial intelligence. Providing highperformance wireless and mobile connectivity to everyone, in an energy-, spectrum- and cost-efficient manner is one of the key challenges of $6 \mathrm{G}$. Thus, strictly speaking, the future $6 \mathrm{G}$ scene will not only be focused on pure communications technologies. Indeed, to efficiently provide ubiquitous wireless connectivity, a great deal of other enabling technologies needs to be developed, as suggested by Figure 1. Even though there is still a long way to go, even to define $6 \mathrm{G}$, based on trends and estimations, there are already some distinctive general characteristics and requirements of $6 \mathrm{G}$ that are highly likely to become certain. Figure 2 depicts some of the most important characteristics and requirements expected for 6G. In a few years from now, as global discussions and exhaustive research take place, precise figures for requirements and characteristic will complement these initial ones.

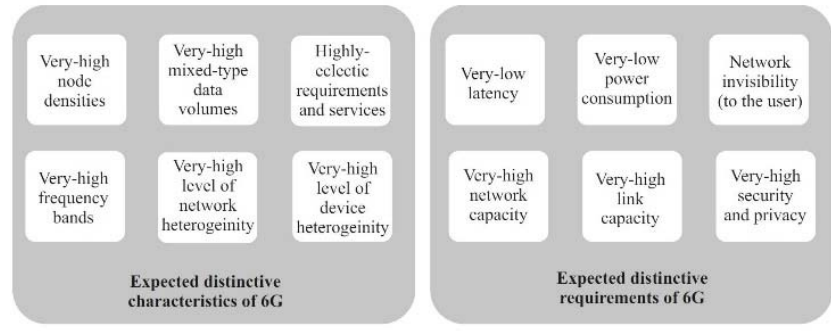

Figure 2. Towards 6G: Expected characteristics and requirements of 6G.

\section{6GENESIS IN A NUTSHELL}

Although our society is already highly connected and currently is being rapidly digitalized, it is expected that this trend will continue. In a not so distant future, we will live in a fully digitalized, hyper-connected and data-driven smart society. Connectivity, one of the key enablers of such world, will be everywhere, unlimited, reliable, with system response practically immediate. Transparent to the user and the society itself, huge amounts of eclectic data will flow through networks, silently helping everyone to have a good life. Such data will be produced by personal and machine communication devices, health monitoring devices, vehicular and sensors, computers and many other sources. How these data will be collected, processed, transmitted, received and ultimately consumed is one of the key issues to be investigated by 6GFP. Inputs, research goals and output of 6GFP are depicted in Fig. 3.

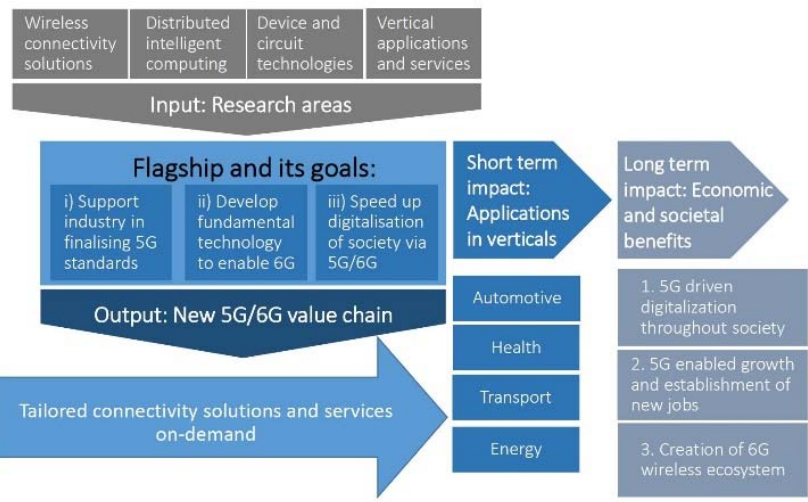

Figure 3. 6Genesis Flagship Program in a nutshell.

Four inter-related core research areas will be studied in great detail by 6GFP to achieve the aforementioned goals. These areas are described next:

\section{A. Wireless Connectivity Solutions and RAN Technologies}

Optimisation of wireless networks makes controlled tradeoffs between data transmission capacity or spectral efficiency and other competing metrics such as energy efficiency and the latency of the service. Thereby a systematic framework and numerical solvers for network design and optimisation can be created.

Network capacity will be enhanced by the introduction of radio spectrum at very high frequency bands, such as mmwave and $\mathrm{THz}$ bands. New solutions for the radio access network (RAN) design and optimisation, together with channel modelling and waveforms, will be developed. New spectrum-sharing mechanisms will allow using opportunistically secondary spectrum to enhance the spectral efficiency.

Physical layer (PHY) technologies including interference coordination/mitigation mechanisms with different types of multiple-input, multiple-output (MIMO) multi-antenna transceiver technologies will be the key enablers in all frequencies. Large arrays and pencil beamforming technologies will be needed at mm-wave and $\mathrm{THz}$ bands to compensate for the path loss in radiowave propagation. For instance, cooperative multipoint (CoMP) processing or its enhanced version of cloud RAN (C-RAN), where clusters perform baseband processing and signal samples are sent to remote radio units (RRUs) via fronthaul links. Novel data modulation approaches with the channel forward error control (FEC) coding and multiplexing will be designed for higher frequencies. Information theory optimisation at the large bandwidth regime with increased phase noise and sparse multipath propagation is crucial.

Network densification and use of dynamic infrastructure are innovative methods to increase spatial spectral efficiency and core technology in 5G. A heterogeneous network (HetNet) with large-coverage macro-cells and local clusters of mainly indoor small cells (on mm-wave bands) is proposed. As base station (BS) density will dramatically increase, BS cost needs to be drastically reduced, and backhaul link capacity need to be increased. The dense 
network configurations will also include dynamic BSs placed in moving nodes such as buses, drones and even vehicles. This will require major breakthroughs in backhauling or fronthauling.

Ultra-reliable low latency communications (URLLC) will be a major difference in $5 \mathrm{G}$ and beyond, compared with $3 \mathrm{G}$ or $4 \mathrm{G}$ systems, where the focus is on the Internet-of-things (IoT). URLLC will enable a whole set of mission-critical applications such as automotive traffic, robotics, real-time control in smart grids, remote surgery, Industry 4.0 and other possible applications. The requirement for lower end-to-end delay (latency) means smaller data packet or frame sizes in practice, as well as flat architectures. Reliable transmission calls for powerful FEC schemes, which are possible only via sufficiently long transmission time or by utilising a large number of parallel diversity channels, making the overall transmission medium more deterministic. Obviously, there is a trade-off between reliability and latency in FEC design which needs to be addressed on physical and networking layers.

Communications concept with embedded positioning may provide an elegant solution to solve the lack of accurate indoor positioning with mm-wave networks. Improved positioning is needed to control moving BSs in dynamic dense networks.

Software-defined networking has the potential to simplify network management. Network Functions Virtualisation (NFV) allows the consolidation of network equipment onto high-volume servers, which could be located in data centres, distributed network nodes and at end-user premises. Network slicing provides an agile and dynamic networking platform on demand, supporting multiple virtual networks on top of a common shared physical infrastructure.

Wireless networks security is essential for the ubiquitous smart society. Security requirements include privacy, authenticity, confidentiality, integrity and availability. Security has to be considered at each individual layer. Wireless transmissions can be jammed and intercepted. Efficient schemes should integrate physical-layer security into existing authentication and cryptography mechanisms for further securing wireless networks.

\section{B. Distributed Intelligent Wireless Computing}

Mobile edge, cloud and fog computing bring intelligence from centralised cloud services closer to users and applications. Mobile edge computing (MEC) refers to data processing power close to the user (called the edge of the network) instead in the cloud or physically at a central computing unit, reducing latency, which is crucial e.g., for smart traffic and smart grids. A mobile cloud or fog computing is a cooperative arrangement of dynamically connected nodes sharing opportunistically their computing resources. Due to heterogeneous service/latency requirements and backhaul/fronthaul limitations, the split of signal-processing functions among cloud, edge servers and radio heads must be optimised in the case of CRAN-type architectures.
Augmented reality (AR)/virtual reality (VR) over wireless is becoming one of the key application drivers for the future. The most promising use cases include interactive story worlds, immersive communication and panoramic video content. The information theory and practical performance requirements from the perspective of human psychology and physiology must be considered. The existing compressiondecompression algorithms may suffice occasionally, but flaws are often noticeable in VR/AR. This justifies considering perception-based coding. Furthermore, it is important to understand how to distribute VR/AR-specific computation across the network at several levels, which is similar to that of a cache hierarchy.

Intelligent distributed computing and data analytics is becoming an inseparable part of wireless networks. Distributed learning mechanisms are needed such that the algorithms are run at BSs, user terminals and IoT devices with limited data, instead of centrally in the core network or with the cloud having access to all source information. Since terminals and devices are not as reliable as high-performance centralised servers, distributed and self-organising schemes are mandatory to provide strong robustness in device and link failures. The protocols must be adaptive and distributed, including distributed communications, routing, load balancing and data caching.

\section{Devices and Circuit Technologies and Implementation}

RF transceiver implementations and integrated circuit (IC) design for mm-wave communications will differ drastically from solutions used at frequencies below $6 \mathrm{GHz}$. High integration can be achieved in RF transceiver design by using advanced silicon nodes, including bulk CMOS, CMOS SOI and SiGe BiCMOS, and careful co-design with off-chip, high-performance antennas based on new materials. One of the major challenges is to find a new balance between the communication concept, digital signal processing and RF technologies where physical and theoretical boundaries of all three areas are thoroughly understood. Digital processing will cause a bottleneck for extremely high data rates, and therefore higher precision RF and analogue signal processing to enable simpler digital solutions is important.

Materials and components determine boundaries for realisation of the complex multi-antenna arrays towards $\mathrm{THz}$ communication systems. Fundamental challenges will occur, including properties of many materials and their fabrication processes above $50 \mathrm{GHz}$. They can influence the intrinsic and extrinsic composition-structure-property relationships, having a more pronounced influence at frequencies where the electromagnetic wavelength approaches the size of the constituents of the materials. For example, the polymers commonly used as substrates of antennas in RF devices have a tendency for increasing losses at tens of $\mathrm{GHz}$, thus degrading the antenna performance. However, according to preliminary research, new nanomaterials and structures enable characteristics for specific applications that can be tailored and optimised in ways not observed previously at other scales. New material opportunities include foams and bio-based, ultra-low permittivity and room-temperature fabricated materials, which can be 3D-printed. Those would 
allow multimaterial integration (even application-tailored substrates) systems that are electrically tuneable and selfhealing.

Embedded systems and software for transceivers and devices must undergo major transitions to meet the URLLC requirements. Future applications need distributed highthroughput local computing nodes and sub-millisecond transport to allow multiple hops without reactivity trade-offs. Latency minimisation alone demands reconsidering the multi-layer protocols resulting in significant overheads, and is also a bandwidth bottleneck. The interplay between transceiver architectures, protocols, latency and bandwidth in turn calls for approaches that pursue reliable communications through highly parallel designs from the wireless path to hardware (HW), and software (SW).

Ubiquitous sensing is an essential technology enabler for intelligent cyber-physical systems critical for future smart societies. The key concepts include 3D range imagining of the environment and machine vision for automated sensing and decision making based on video-captured information. A $3 \mathrm{D}$ range imager is a device that can measure the shape and location of targets within its field of view and has huge potential in e.g., vehicle control and guidance systems, drones, robotics, smart home, security, man-machine communication, gesture control and consumer electronics. These applications require a relatively high measurement rate, low cost and realisation in miniature comparable to that found in modern CMOS cameras. In contrast to $3 \mathrm{D}$ imagining, machine vision research concentrates on developing novel methods and solutions for computer vision and signal analysis, with applications including affective computing, human-computer interaction, industrial and biomedical purposes. These are all key methodological components in the perceptual interfacing IoT scenarios, heavily relying on machine learning and AI. The capability of state-of-the-art approaches in-the-wild is a major research topic, e.g. how to capture more information from human faces and expressions than humans are capable of distinguishing in everyday situations and environments.

\section{Vertical Applications and Services}

Vertical applications are strongly driving the development of future communications systems. We envision vertical specific optimized technical solutions as well as network operating models to appear in the $6 \mathrm{G}$ era. According to the 5G Infrastructure Association of 5G-PPP, the main verticals adopting upcoming wireless enablers include factories of the future (Industry 4.0), automotive and transportation, energy, media and entertainment, and e-health. Verticals also efficiently steer the research of different teams towards commonly defined goals and provide the necessary multidisciplinary approach to research. In terms of verticals, healthcare is expected to have a high priority, with ubiquitous $24 / 7$ preventive health enabled by smart sensor environments and future connectivity in focus. Industry 4.0 vertical, factory floor situational awareness with URLLC connectivity is of great interest. In the transportation vertical, autonomous vehicles that would be considered autonomous agents sensing the environment and communicating with other vehicles over low latency links is the setting for research.

An experimental wireless network is an essential tool to bring research findings to real-life tests in co-operation with businesses and society at large. This is particularly important as the scope of the Flagship is broad, covering radio transmission and transceivers, new component technology and materials, radio access networks, storage and distributed computing in wireless networks and inclusion of novel sensory and visualisation tools. 5G/6G Test Network is an efficient tool to steer the research of both radios and applications towards common goals in different verticals. Thus, it is instrumental in guaranteeing success of 6 Genesis. This setup is necessary to address the societal and business challenges besides the scientific objectives set for research.

Techno-economic and business considerations are very important for alternative deployment scenarios of future network technologies. Network densification, spectrum sharing and the increasing amounts and diversifying nature of data will call for fundamentally new and innovative network operator and ownership models. This development raises three sets of questions: a) Who owns the networks and has incentives in making investments in network infrastructure? b) Who owns the radio spectrum and how can it be utilised more dynamically in an affordable manner? c) Who owns the data and what added value can be generated using the data in mobile networks? Answers to these questions might require important changes in telecommunications legislation, mobile communication market structures and network technologies. How different network ownership and service provisioning models affect the design of radio access systems needs to be understood to guarantee success for the organic or holistic growth of future digital services.

\section{RESEARCH IMPLEMENTATION}

Research will be carried out following a number of research lines, including information and communications theory, implementation and proof-of-concept of $6 \mathrm{G}$ technologies and test network, and others. Details of these research lines are briefly provided below. A short presentation of the research consortium is also introduced in this section. The overall Flagship programme logic and implementation are illustrated in Figure 4.

\section{A. Technology Enablers towards $6 G$}

Information \& communications theory \& optimisation for mm-wave and (sub-)THz bands: Develop fundamental information and communications theory using the physicsinspired models of materials, circuits and wave propagation to enable the basic design tools and guidelines for RAN design and optimisation. These will be realised as a systematic optimisation framework enabling different design targets.

Superfast wireless broadband connectivity: Use the optimisation framework to provide solutions for high-rate and capacity technologies in the Tbps range, with emphasis on energy-efficient solutions. The work will also consider the transceiver RF and $\mathrm{BB}$ requirements and their CRAN-based architectures and basic technology options, in addition to RAN algorithms and methodology. 


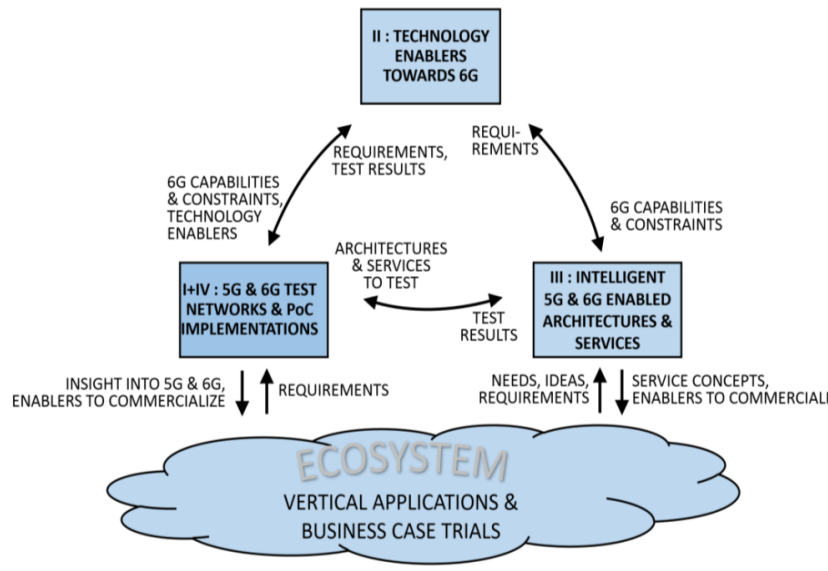

Figure 4. 6GFP logic and implementation.

Ultra low latency communications: Develop the optimisation framework to allow the network to support low latencies with high reliability at the cost of compromised spectral efficiency such that RAN protocols and transceivers can be specified. System architectures and the limits of CRAN and other realisations will be addressed to specify costand energy-efficient realisations.

Security for 6G: Develop a thorough security analysis and solution framework for the new RAN technology family on PHY and higher layers. Network-level software security aspects will be considered and integrated in the RAN and SDN concepts.

THz technology enablers towards 6G: Create sub-THz band wireless transceivers capitalising the expertise on mmwave devices and sub-THz imaging circuits as $6 \mathrm{G}$ enablers with related channel and propagation modelling, system and communications theory. Develop new low-loss materials and packaging solutions for adequate system performance.

High-risk technology enablers towards 6G: Carry out active scouting on promising and relevant developments for the 2030 era for innovativeness and risk taking for research. As an example, ground-breaking developments in quantum theory and quantum communications will be monitored and feasibility studies will be launched whenever meaningful.

\section{B. Implementation of $6 G$ Technologies and Test Network}

Technologies towards $6 \mathrm{G}$ communications are still at a very early phase of maturity. Solutions to fully exploit the capacity theoretically provided at frequencies reaching $100 \mathrm{GHz}$ and beyond also need proof-of-concept realisations before they can be adopted as part of $6 \mathrm{G}$ network trials. Extremely fast radio links together with advanced sensing options at $\mathrm{THz}$ frequencies will provide a base to build key new capabilities towards $6 \mathrm{G}$, thus leading concept development to take physical constraints into account more carefully than ever before. Fundamentally new innovations that need demonstrations at system level include the feasibility of very large-scale and efficient (low loss) antenna arrays to provide sufficient antenna apertures for decent range, processing of very wide bandwidths available at sub- $\mathrm{THz}$ and $\mathrm{THz}$ region, new opportunities in $\mathrm{THz}$ sensing, and power generation at sub- $\mathrm{THz}$ and $\mathrm{THz}$ frequencies that cannot be performed as at lower frequencies due to speed limitations of transistor technologies and thus the maximum available operating frequency. The current network architecture, see Figure 5, uses technologies including 3GPP specified evolved packet core elements and the latest LTE radio access technology at 2.6 and $3.5 \mathrm{GHz}$, with special emphasis on small cell-based solutions. The first $5 \mathrm{G}$ proof-of-concept devices at $28 \mathrm{GHz}$ are also an integral part of the network, which contains close to 500 sensors, two outdoor macro base stations and 15 indoor small cells. The network is complemented by big data computing servers for analytics purposes for the data collected via sensors and broadband radios. Some of these servers are distributed within the network, thus allowing mobile edge computing and caching services. The 5GTN network is demonstrated in several vertical domains already, including eHealth and digital factory.

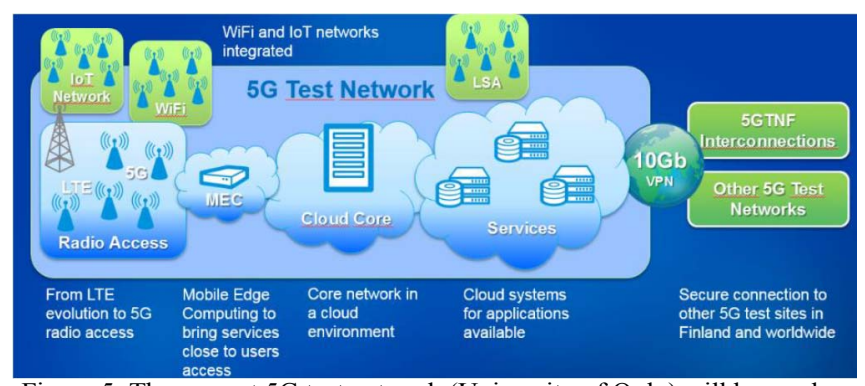

Figure 5. The current 5G test network (University of Oulu) will be used as the starting point for developing the proof-of-concept for $6 \mathrm{G}$ enabling technologies.

\section{Research Collaboration: The 6GFP Consortium}

The 6GFP consortium consists of academia, research centers, industrial partners (from small to large-size), business units and others. Centre for Wireless Communications (CWC) from University of Oulu, is the coordinator of the initiative. The chief collaboration partners of 6GFP consortium are listed below. Other partners may also join the initiative as the program progresses.

- University of Oulu, Finland

- Consortium led by Centre for Wireless Communications, University of Oulu, Finland.

- VTT Technical Research Centre of Finland Ltd

- Aalto University, Finland

- Oulu University of Applied Sciences (OUAS)

- Nokia and Nokia Bell Labs

- BusinessOulu (BO), Finland

- Ecosystem partner: 5G Test Network Project Consortium (5GTN+)

\section{CONCLUSION}

This paper presents the visions and research plans of the newly created consortium 6GFP, aiming to ultimately develop sound concepts and enabling technologies for the upcoming 6G. The 6GFP is an eight-year research initiative of a consortium formed by leading Finnish academic and industrial partners, aiming at developing, implementing and testing key enabling technologies for $6 \mathrm{G}$. The paper first presented some visions, trends and rough expectations for the upcoming $6 \mathrm{G}$. In this program, $6 \mathrm{G}$ is investigated from a wide and realistic perspective, considering not only the communicational part of it, but also looking into other highly relevant parts, such as computer science, engineering, 
electronics and material science. This integral approach is instrumental to achieve truly novel and highly efficient solutions. Important technical topics are highlighted and briefly justified, to help the reader to better understand their inclusion in this program. The paper also considers implementation aspects of the program, including some promising technologies as well as ideas for realizing evaluation and test of the developed solutions using an existing state-of-the-art $5 \mathrm{G}$ test network.

\section{ACKNOWLEDGMENT}

This research has been financially supported by Academy of Finland 6Genesis Flagship (grant 318927)

\section{REFERENCES}

[1] European Commission, "Strategic spectrum roadmap towards 5G for Europe: RSPG second opinion on 5G networks," Radio Specrum Policy Group (RSPG), RSPG18-005, 2018.

[2] ITU-R. (2015). IMT Vision - framework and overall objectives of the future development of IMT for 2020 and beyond" ITU-R Report M. 2083. International Telecommunications Union.

[3] Agiwal, M., Roy, A., \& Saxena, N. (2016). Next generation 5G wireless networks: A comprehensive survey. IEEE Communications Surveys \& Tutorials, 18, 1617-1655.

[4] S. Parkvall, E. Dahlman, A. Furuskär and M. Frenne, "NR: The new 5G radio access technology", IEEE Communications Standards Magazine, vol. 1, no. 4, pp. 24-30, Dec. 2017.

[5] M. Matinmikko, M. Latva-aho, P. Ahokangas, and V. Seppänen, "On regulations for 5G: Micro licensing for locally operated networks", Telecommunications Policy, vol. 42, no. 8, pp. 622-635, Sept. 2018. .

[6] M. Patzold, "5G Readiness on the Horizon [Mobile Radio]," in IEEE Vehicular Technology Magazine, vol. 13, no. 1, pp. 6-13, March 2018.

[7] G. Durisi, T. Koch and P. Popovski, "Toward Massive, Ultrareliable, and Low-Latency Wireless Communication With Short Packets," in Proceedings of the IEEE, vol. 104, no. 9, pp. 1711-1726, Sept. 2016.

[8] F. Boccardi, R. W. Heath, A. Lozano, T. L. Marzetta and P. Popovski, "Five disruptive technology directions for 5G," in IEEE Communications Magazine, vol. 52, no. 2, pp. 74-80, February 2014.

[9] http://www.oulu.fi/university/6gflagship 